antarctic continent. His own discoveries in the Australian province confirm the belief, previously held, of the continuity of the southern land mass. The rocks are pre-Cambrian and early Palæozoic in the main but on the Pacific side there is a large tract of Permian to Triassic beds lying almost horizontally on the older formations. South of the American continent, however, a region of different tectonic structure is found with folded beds of secondary and tertiary age. This region, known generally as Graham Land, appears to be an island chain bound together and to the continent by a permanent shelf-ice sheet of great thickness. Sir Douglas Mawson pointed to the possibility of the Ross Barrier at the head of the Ross Sea extending unbroken to the barrier at the head of the Weddell Sea. If this is the case, the archipelago of Graham Land is not part of the antarctic continent but is joined to it by a vast sheet of floating shelf-ice. The lecture has recently been made available in pamphlet form.

\section{Treatment of Pernicious Anæmia}

THE treatment of pernicious anæmia by a diet containing large amounts of liver or by the oral administration of an extract of liver or of desiccated stomach tissue is now the standard treatment for this disease. For patients who are severely ill and cannot tolerate oral administration, the intramuscular or intravenous injection of a potent extract of liver may be a life-saving measure; moreover, by the parenteral route, smaller doses are required and the response of the blood is quicker. The British Drug Houses, Ltd., London, N.1, are now issuing an extract of liver for intramuscular injection. Each cubic centimetre contains the anti-anæemic principles of $50 \mathrm{gm}$. of fresh liver, whilst its depressor effect does not exceed that of a 0.0005 per cent solution of histamine acid phosphate when introduced by the intravenous route into an etherised ca.t. In addition to Liver Extract B.D.H., and Liquid Liver Extract B.D.H., preparations for oral administration in the treatment of pernicious anæmia, the British Drug Houses also issue the product 'Livogen', which is a highly concentrated liquid preparation containing in one fluid ounce the whole of the hæmotopoietic principles (including vitamin B) of four ounces of fresh liver. It contains additional vitamin $\mathrm{B}$ extract equivalent to one ounce of fresh yeast in each fluid ounce, together with five grains of hæmoglobin. For the specific anæmias, in which massive doses of iron are indicated, iron ammonium citrate may be dispensed with Livogen, the two products being in every way compatible. Livogen is recommended for use in convalescence from severe illness as well as in both primary and secondary anæmias.

\section{Odour of Vulcanised Rubber}

As the rather unpleasant odour of vulcanised rubber limits its applications, the Rubber Growers' Association has instituted an investigation into its causes and prevention (Bull. Rubber Growers' Association, May, 1933). When rubber goods were made from inferior grades of wild rubber, their smell was due to putrefactive changes, but in these days of plantation rubber, the trouble arises mainly from the accelerator employed, although macintoshes and other proofed goods owe their smell to the low-grade petroleum and coal-tar naphtha products that are used as diluents when the rubber is manufactured. Vulcanised goods can often be temporarily freed from odour by steaming them, but permanent freedom can only be secured by removing the substances that give rise to odour, whether they be constituents of the raw rubber or added to it in the course of manufacture. The smell due to accelerators may be of the 'sulphide' or of the 'amino' type, according as they contain sulphur or amino-nitrogen. So far, no good accelerator that is free from these constituents has been found, but Messrs. H. P. Stevens and E. J. Parry, the chemists in charge of the investigation, have discovered that the presence of zinc carbonate in the compounding mixture reduces the smell to a minimum. The slight 'amino' smell characteristic of the unvulcanised plantation rubber has been traced by them to the proteins, and not to the resins, present in the raw material; and it can be practically eliminated if the latex is purified by such methods as digestion with dilute caustic, centrifuging, creaming, ultra-filtration, or dialysis. When the problem has been definitely solved, it will be possible to use vulcanised rubber for lining foodcontainers and brewers' vats, and in the preparation or packing of foodstuffs in general.

\section{Heating of Domestic Pendant Lamp Fittings}

The usefulness of scientific research on everyday domestic devices is shown by a paper read to the Institution of Electrical Engineers by P. D. Morgan, H. G. Taylor and W. Lethersich on April 20. The problem they had to solve was to find the best way of keeping the heating of lamp fittings and their connecting flexible wires at such a low value that the rubber insulation would not deteriorate. This deterioration takes the form of hardening and making brittle the rubber surrounding the wire. In this case a slight disturbance may cause it to crack off and expose the bare conductor. This may lead to a short-circuit and so possibly to igniting the 'flex'. It would be a rare occurrence but it would be advisable to prevent it. The deterioration of the rubber is due to its oxidisation, which is accelerated by heat. The introduction of gas-filled lamps has appreciably raised the temperature of the flexible wire connected to the holder and thus the problem has become more urgent.

The solution adopted in the United States is to use flexible cords capable of withstanding higher working temperatures than the usual standard rise of $23 \cdot 4^{\circ} \mathrm{C}$. at a distance of $\frac{5}{8} \mathrm{in}$. from the terminal. A lampholder and its connecting leads receive heat by conduction through the cap from the hot gas in the lainp and dissipate it by radiation and convection. It is necessary to make the heat conducted through the cap of the lamp smaller and facilitate the 